\title{
Editorial
}

\section{Discrete Dynamics of Fractional Systems: Theory and Numerical Techniques}

\author{
Jorge E. Macías-Díaz $\mathbb{D},{ }^{1}$ Qin Sheng $\mathbb{D},{ }^{2}$ and Stefania Tomasiello $\mathbb{D}^{3}$ \\ ${ }^{1}$ Departamento de Matemáticas y Física, Universidad Autónoma de Aguascalientes, 20100 Aguascalientes, AGS, Mexico \\ ${ }^{2}$ Department of Mathematics, Baylor University, Waco, TX 76798-7328, USA \\ ${ }^{3}$ Consorzio di Ricerca Sistemi ad Agenti, Università degli Studi di Salerno, Fisciano, 84084 Salerno, Italy
}

Correspondence should be addressed to Jorge E. Macías-Díaz; jemacias@correo.uaa.mx

Received 27 February 2018; Accepted 28 February 2018; Published 24 April 2018

Copyright (c) 2018 Jorge E. Macías-Díaz et al. This is an open access article distributed under the Creative Commons Attribution License, which permits unrestricted use, distribution, and reproduction in any medium, provided the original work is properly cited.

In recent decades, fractional calculus has found a large number of profound applications, which have triggered the development of both the theory and methods for more reliable discretization and approximations of the dynamics of continuous systems. Fractional order models and discretized problems are nonlocal. They provide better descriptions of and, ultimately, deeper insights into underlying complex phenomena in sciences and technologies. Novel new analytical approaches have become a key to the study of qualitative properties of the aforementioned fractional systems and the existence and uniqueness of their nonlocal solutions.

Continuous models based on systems of ordinary or partial differential equations have been investigated under proper criteria of discretization in this special issue. Novel numerical approximations of solutions of fractional systems are also investigated. In fact, the search for discrete techniques which are faster and stable, that possess higher orders of convergence at lower computational costs, and that preserve the main features of the solutions of interest has been a constant pursuit in numerical analysis. To this end, this special issue pays a special attention to the discretization of continuous fractional systems that preserve important characteristics including the positivity, boundedness, convexity, monotonicity, and energy of the underlying systems.

The 8 research papers in this special issue are highly selective. These high-quality papers represent the latest developments in the theory of discrete fractional systems and the discretization of fractional differential equations arising from sciences and technologies. The final contributed papers focus on issues like

(i) Lebesgue- $p$ norm convergence analysis of $\mathrm{PD}^{\alpha}$-type iterative learning control for fractional order nonlinear systems,

(ii) solution existence for initial-value problems of hybrid fractional sum-difference equations,

(iii) homotopy series solutions to time-space fractional coupled systems,

(iv) numerical simulations of one-dimensional fractional nonsteady heat transfer models based on the second kind Chebyshev wavelet,

(v) numerical analysis of fractional order epidemic models of childhood diseases,

(vi) weak solutions for partial random Hadamard fractional integral equations with multiple delays,

(vii) modified function projective synchronization for a partially linear and fractional order financial chaotic system with uncertain parameters,

(viii) two new approximations for variable order fractional derivatives.

Jorge E. Macías-Díaz Qin Sheng

Stefania Tomasiello 


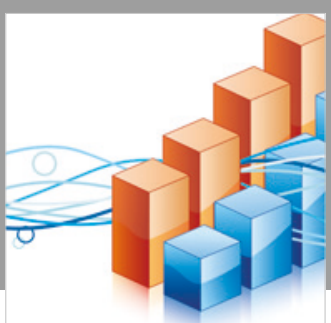

Advances in

Operations Research

\section{-n-m}
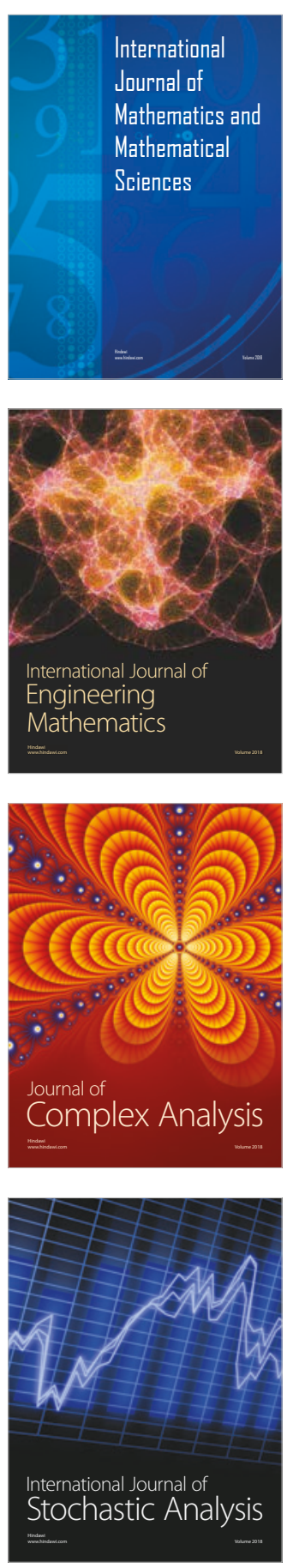
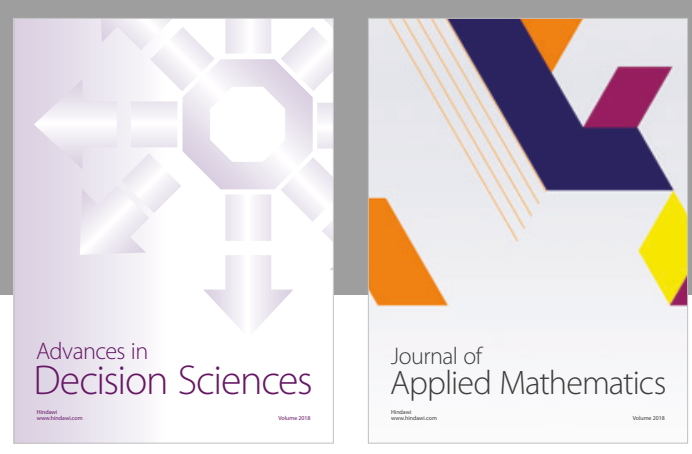

Journal of

Applied Mathematics
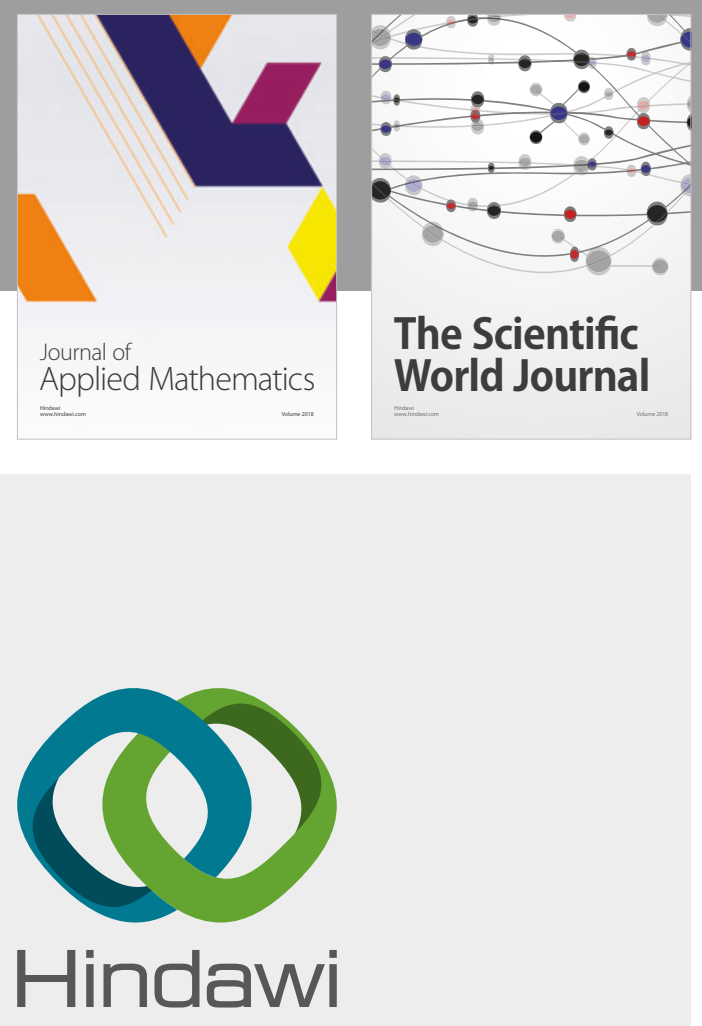

Submit your manuscripts at

www.hindawi.com

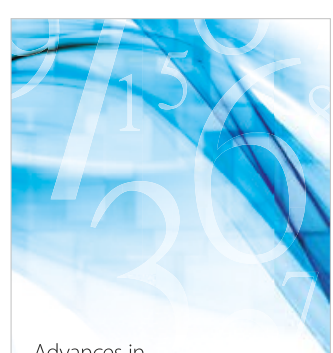

Advances in
Numerical Analysis
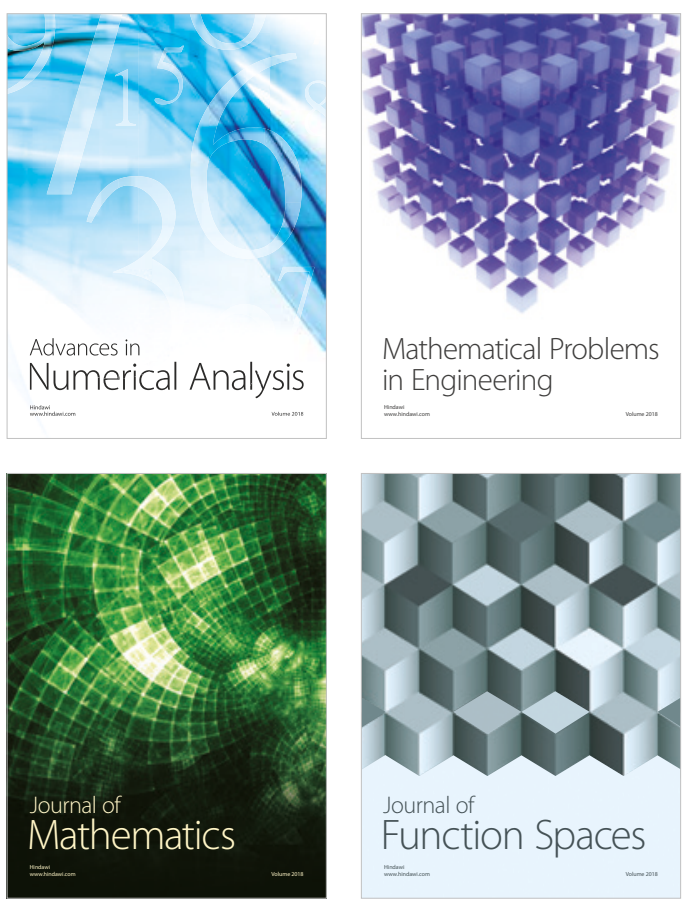

Mathematical Problems in Engineering

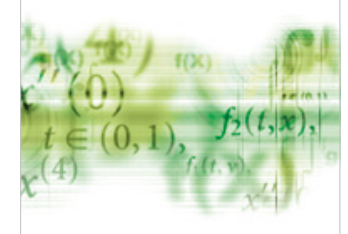

International Journal of

Differential Equations

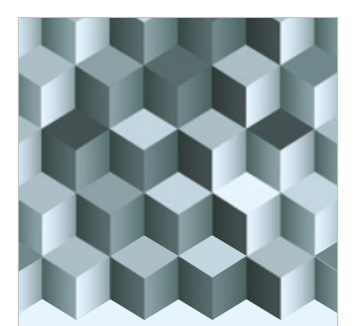

Journal of

Function Spaces

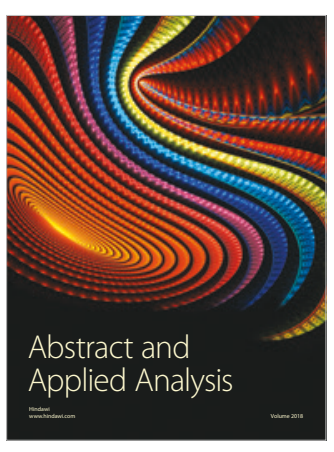

The Scientific

World Journal

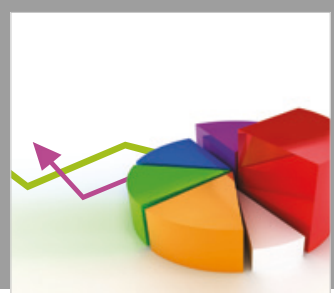

Journal of

Probability and Statistics
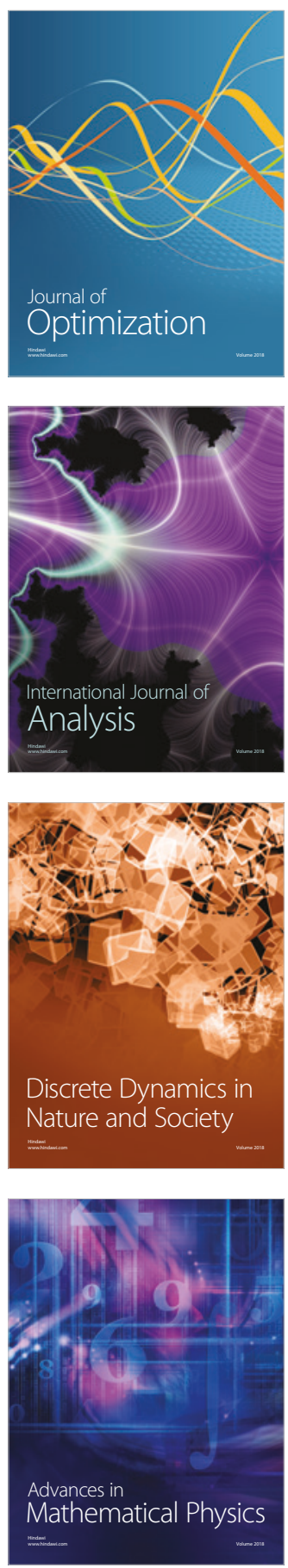